Published in final edited form as:

Clin Gastroenterol Hepatol. 2019 July ; 17(8): 1561-1570.e3. doi:10.1016/j.cgh.2018.11.036.

\title{
Association Between Intake of Red and Processed Meat and Survival in Patients With Colorectal Cancer in a Pooled Analysis
}

\author{
Prudence R Carr ${ }^{1}$, Barbara Banbury ${ }^{2}$, Sonja I Berndt ${ }^{3}$, Peter T CampbelI ${ }^{4}$, Jenny Chang- \\ Claude $^{5,6}$, Richard B Hayes ${ }^{7}$, Barbara V Howard ${ }^{8}$, Lina Jansen ${ }^{1}$, Eric Jacobs ${ }^{9}$, Dorothy S \\ Lane $^{10}$, Reiko Nishihara ${ }^{11}$, Shuji Ogino ${ }^{11,12,13,14}$, Amanda I Phipps ${ }^{15}$, Martha L Slattery ${ }^{16}$, \\ Marcia L Stefanick $^{17}$, Robert Wallace ${ }^{18}$, Viola Walter ${ }^{1}$, Emily White ${ }^{2,15}$, Kana Wu ${ }^{19}$, Ulrike \\ Peters $^{2,20}$, Andrew T Chan ${ }^{21,22}$, Polly A Newcomb ${ }^{2,15}$, Hermann Brenner ${ }^{1,23,24}$, and Michael \\ Hoffmeister ${ }^{1}$ \\ ${ }^{1}$ Division of Clinical Epidemiology and Aging Research, German Cancer Research Center \\ (DKFZ), Heidelberg, 69120 Germany \\ ${ }^{2}$ Public Health Sciences Division, Fred Hutchinson Cancer Research Center, Seattle, WA 98109, \\ USA
}

${ }^{3}$ Division of Cancer Epidemiology and Genetics, National Cancer Institute, National Institutes of Health, Bethesda, MD 20892, USA

${ }^{4}$ Epidemiology Research Program, American Cancer Society, Atlanta, GA 30303, USA

${ }^{5}$ Division of Cancer Epidemiology, German Cancer Research Center (DKFZ), Heidelberg, 69120 Germany

${ }^{6}$ Genetic Tumour Epidemiology Group, University Medical Center HamburgEppendorf, University Cancer Center Hamburg, Hamburg, 20246 Germany

${ }^{7}$ Division of Epidemiology, Department of Population Health, New York University School of Medicine, New York, NY 10016, USA

${ }^{8}$ Department of Medicine, Georgetown University, Washington D.C. 20057, USA

${ }^{9}$ American Cancer Society, Atlanta, GA 30303, USA

\footnotetext{
Corresponding author: Michael Hoffmeister, PhD, Division of Clinical Epidemiology and Aging Research, German Cancer Research Center (DKFZ), Im Neuenheimer Feld 581, 69120 Heidelberg, Germany, Phone +49 6221 42-1303,

m.hoffmeister@dkfz.De.

Author contributions:

Study concept and design: SIB, PTC, JCC, RBH, BVH, EJ, DSL, RN, SO, AIP, MLSl, MLSt, RW, EW, KW, UP, ATC, PAN, HB, MH; acquisition of data: SIB, PTC, JCC, RBH, LJ, BVH, EJ, DSL, RN, SO, AIP, MLSl, MLSt, RW, VW, EW, KW, UP, ATC, PAN, $\mathrm{HB}, \mathrm{MH}$; analysis and interpretation of data: $\mathrm{BB}, \mathrm{PRC}, \mathrm{MH}$; drafting of the manuscript: PRC; critical revision of the manuscript for important intellectual content: all authors; statistical analysis: BB; obtained funding: SIB, PTC, JCC, RBH, BVH, EJ, DSL, RN, SO, AIP, MLSl, MLSt, RW, EW, KW, UP, ATC, PAN, HB, MH; study supervision: SIB, PTC, JCC, RBH, BVH, EJ, DSL, RN, SO, AIP, MLS, MLS, RW, EW, KW, UP, ATC, PAN, HB, MH. All authors read and approved the final manuscript.

Publisher's Disclaimer: This is a PDF file of an unedited manuscript that has been accepted for publication. As a service to our customers we are providing this early version of the manuscript. The manuscript will undergo copyediting, typesetting, and review of the resulting proof before it is published in its final citable form. Please note that during the production process errors may be discovered which could affect the content, and all legal disclaimers that apply to the journal pertain.

Conflict of interest: The authors declare that they have no conflict of interest.
} 
${ }^{10}$ Department of Family, Population and Preventive Medicine, Stony Brook School of Medicine, NY 11790, USA

${ }^{11}$ Program in MPE Molecular Pathological Epidemiology, Department of Pathology, Brigham and Women's Hospital, and Harvard Medical School, Boston, MA 02215, USA

${ }^{12}$ Department of Oncologic Pathology, Dana-Farber Cancer Institute, Boston, MA 02215, USA

${ }^{13}$ Broad Institute of MIT and Harvard, Cambridge, MA, USA

${ }^{14}$ Department of Epidemiology, Harvard T.H Chan School of Public Health, Boston, MA 02215, USA

${ }^{15}$ Cancer Prevention Program, Fred Hutchinson Cancer Research Center, Seattle, WA 98109, USA

${ }^{16}$ Department of Internal Medicine, University of Utah Health Sciences Center, Salt Lake City, UT 84132, USA

${ }^{17}$ Stanford Prevention Research Center, Stanford University School of Medicine, Stanford, CA 94305-5411, USA

${ }^{18}$ Department of Epidemiology, The University of lowa, lowa City, IA 52242, USA

${ }^{19}$ Department of Nutrition, Harvard T.H Chan School of Public Health, Boston, MA 02115, USA

${ }^{20}$ Department of Epidemiology, University of Washington School of Public Health, Seattle, WA 98195, USA

${ }^{21}$ Division of Gastroenterology, Massachusetts General Hospital, Boston, MA 02114, USA

${ }^{22}$ Channing Division of Network Medicine, Department of Medicine, Brigham and Women's Hospital and Harvard Medical School, Boston, MA 02115, USA

${ }^{23}$ Division of Preventive Oncology, German Cancer Research Center (DKFZ), Heidelberg, 69120 Germany

${ }^{24}$ German Cancer Consortium (DKTK), German Cancer Research Center (DKFZ), Heidelberg, 69120 Germany

\section{Abstract}

Background \& Aims: Red and processed meat intake is associated with colorectal cancer (CRC) incidence, but it is not clear if intake is associated with patient survival after diagnosis

Methods: We pooled data from 7627 patients with stage I-IV CRC from 10 studies in the International Survival Analysis in Colorectal Cancer Consortium. Cox proportional hazards regression models were used to evaluate the associations of intake of red and processed meat before diagnosis with overall and CRC-specific survival.

Results: Among 7627 patients with CRC, 2338 died, including 1576 from CRC, over a median follow-up time of 5.1 years. In multivariable-adjusted analyses, higher intake of red or processed meat was not associated with overall survival of patients with stage I-III CRC: Q4 vs Q1 red meat hazard ratio [HR], 1.08 (95\% CI, 0.93-1.26) and Q4 vs Q1 processed meat HR, 1.10 (95\% CI, 0.93-1.32) or with CRC-specific survival: Q4 vs Q1 red meat HR, 1.09 (95\% CI, 0.89-1.33) and 
Q4 vs Q1 processed meat HR, 1.11 (95\% CI, 0.87-1.42). Results were similar for patients with stage IV CRC. However, patients with stage I-III CRC who reported an intake of processed meat above the study-specific medians had a higher risk of death from any cause (HR, 1.12; 95\% CI, 1.01-1.25) than patients who reported eating at or less than the median.

Conclusion: In this large consortium of CRC patient cohorts, intake of red and processed meat before a diagnosis of CRC was not associated with shorter survival time after diagnosis, although a possible weak adverse association cannot be excluded. Studies that evaluate dietary data from several time points before and after cancer diagnosis are required to confirm these findings.

\section{Keywords}

ISACC; colon cancer; epidemiology; risk factor

\section{Introduction}

The role of diet and lifestyle factors in colorectal cancer (CRC) has been extensively studied and evidence suggests that red and processed meat intake is an important dietary risk factor $^{1,2}$ Meta-analyses have concluded that high intake of red and processed meat is associated with increased risk of colorectal, colon and rectal cancers ${ }^{1,3,4}$, and in 2015 the International Agency for Research on Cancer classified processed meat as carcinogenic to humans and red meat as probably carcinogenic to humans ${ }^{2}$. The World Cancer Research Fund/American Institute for Cancer Research (WCRF/AICR) in 2017 judged red meat to be a probable risk factor for $\mathrm{CRC}$, while processed meat was judged to be a convincing risk factor for CRC, recommending to limit the intake of red meat and to avoid processed meat ${ }^{1}$.

However, in contrast, limited evidence exists on the role of red and processed meat intake in survival after a diagnosis of $\mathrm{CRC}^{5,6}$. To our knowledge, few studies have investigated the association between meat consumption and overall survival among men and women diagnosed with CRC ${ }^{7-12}$ and results are inconclusive. In two studies, pre-diagnostic red and processed meat intake was not associated with overall survival or CRC-specific survival $^{11,12}$. In another study, higher red and processed meat intake was associated with all-cause mortality and CVD-specific mortality, but not with CRC-specific mortality ${ }^{8}$. Two other studies which assessed the associations between pre-diagnostic meat intake (not limited to red and processed meat) and overall survival did not observe any associations ${ }^{7,10}$.

Hence, using individual patient data in ten observational epidemiological studies of the International Survival Analysis in Colorectal Cancer Consortium (ISACC), we aimed to evaluate the association of pre-diagnostic red meat and processed meat intake with overall and CRC-specific survival.

\section{Methods}

\section{Study population}

This analysis included 10 studies from the International Survival Analysis in Colorectal Cancer Consortium (ISACC). We included data from 7 prospective U.S cohort studies [Cancer Prevention Study II Nutrition cohort (CPSII) ${ }^{13}$; Health Professionals Follow-up 
Study (HPFS) ${ }^{14}$; Nurses' Health Study I (NHS I) ${ }^{15-17}$; Physician's Health Study (PHS) ${ }^{18}$; Prostate, Lung, Colorectal and Ovarian Cancer Screening Trial (PLCO) ${ }^{19}$, 20; VITamins And Lifestyle Study (VITAL) ${ }^{21}$ and Woman's Health Initiative (WHI) ${ }^{22}$ and 3 population based case-control studies from the US and Germany with follow-up of patients [Darmkrebs: Chancen der Verhuetung durch Screening (DACHS) ${ }^{23}$, 24; Diet, Activity and Lifestyle Survey (DALS) ${ }^{25}$ and PostMenopausal Hormone Study within the Colorectal Cancer Family Registry (PMH- CCFR)]. These studies are also included in the Genetics and Epidemiology of Colorectal Cancer Consortium (GECCO) which was primarily responsible for the data harmonization ${ }^{26,27}$ All CRC cases were confirmed by medical record, pathology report or death certificate and included follow-up for survival. Informed consent was obtained from all participants, and the studies were approved by their respective institutional review board.

\section{Data collection and follow-up}

Information on demographic and lifestyle factors was collected by self-report using in person interviews and/or structured questionnaires as detailed previously ${ }^{28}$. As previously described, a multistep data harmonization procedure was carried out to combine the data across the studies for pooled analyses ${ }^{28}$. Red meat intake (unprocessed red meat including processed red meat, i.e. sausages, in some studies) and processed meat (processed red meat and processed white meat) intake from all participating studies was classified as number of servings per day. Other variables collected by the studies included: age at diagnosis, sex, body mass index (BMI) $\left(\mathrm{kg} / \mathrm{m}^{2}\right.$ ), smoking status (ever/never at reference time), non-steroidal anti-inflammatory drug (NSAID) use including aspirin (yes/no for regular use at reference time), education ( $<$ high school graduate, high school graduate, some college or technical school, college graduate or graduate degree), alcohol intake (nondrinker, 1-28 g/day, $>28 \mathrm{~g} /$ day), family history of CRC in a first degree relative (yes, no), history of diabetes (yes, no), and physical activity (yes: vigorous and moderate physical activity $\geq 1$ hour/week, no: vigorous and moderate physical activity $<1$ hour/week). Additional dietary variables included dietary intake of calcium, folate, fiber, fruit, and vegetables. Information on cancer site (proximal colon, distal colon, or rectal) and cancer stage as determined by AJCC or SEER (AJCC stage 1 or SEER local, AJCC stage 2/3 or SEER regional, AJCC stage 4 or SEER distant) was available for all studies.

The details of assessing survival in the included studies have been described previously 13, 14, 17, 21, 23, 25, 29-32 Briefly, half of the studies used active follow-up to ascertain vital status (HPFS, NHS, PHS, PLCO, and WHI); dates and cause of death were confirmed via review of death certificates and/or medical records. For the other studies (VITAL, CPS-II, DACHS, DALS, PMH-CCFR), vital status was determined through linkage to the National Death Index, state cancer registries, state death records, or population registries with cause of death verified by death certificates. In all studies, patients alive at the most recent followup or data linkage were censored on that date in the survival analysis.

\section{Statistical analysis}

All statistical analyses were performed at the central ISACC coordinating center using the individual level harmonized data pooled across studies. We used Cox proportional hazards 
regression to calculate hazard ratios (HRs) and 95\% confidence intervals (CI) for the association of baseline red meat and processed meat intake with overall and CRC-specific survival. Survival time was calculated as the time from diagnosis of CRC to death or end of follow-up. In analyses of CRC- specific survival, patients who died from causes other than CRC were censored at the time of death. We decided a priori to stratify results for patients by disease stage analyzing stages I-III and stage IV separately because the overall survival for stage IV patients is poor. Median follow-up time was calculated based on the Kaplan Meier estimate of potential follow-up ${ }^{33}$

We constructed separate models for overall and CRC-specific survival. A combination of forwards and backwards variable selection was used to determine variables that were significantly associated with survival for inclusion in the multivariable adjusted model. All models included age at diagnosis, sex, cancer stage, cancer site and study. The multivariable model included additionally: BMI, smoking status, family history of CRC, aspirin/NSAID use and education. Other potential confounding variables that were considered but not included in the models were: physical activity, history of diabetes, total energy intake, alcohol intake, and intake of calcium, folate, fruit, vegetables and fiber. We examined Schoenfeld residuals and included strata variables for those which violated the proportional hazards assumption. Missing data for BMI, smoking status, family history of CRC, regular use of aspirin/NSAID use and education (all less than 8\%) were accounted for by mean and mode imputation, and a missing indicator variable was used for missing stage at diagnosis.

We used study- and sex-specific quartiles of red meat and processed meat intake, using the lowest quartile as the referent category. We also examined the association with red meat and processed meat intake modelled as a dichotomous variable ( $\zeta \mathrm{or}>$ study-specific medians) and as a continuous variable (servings/day) (see Supplementary Table 1 for study-specific median intake). Tests for trend across quartiles were conducted by assigning participants the study and sex specific median value per quartile. We also conducted analyses stratified by age ( $<70$ years, $>70$ years), sex (male, female), cancer site (colon, rectum), family history of CRC (yes, no), BMI (18.5-24.9, >24.9-<30, >30) and study design (cohort, case-control). We tested the significance of multiplicative interaction with age, sex, family history and BMI using the likelihood ratio tests. All analyses were performed using R 2.15.3.

\section{Results}

From 10 studies, data were harmonized for a total of 8316 (4502 female, 3814 male) patients. We excluded individuals from the present analyses with missing information on red meat $(n=689)$ or processed meat $(n=968)$ and those with missing follow up time $(n=2)$. Consequently, the total study population for the current analyses comprised of $7625 \mathrm{CRC}$ patients: 6695 CRC patients with stage I-III disease and 930 CRC patients with stage IV disease. More information on the individual studies is provided in Supplementary Table 1. Among the patients with stage I-IV CRC included in the analyses, 2338 (31\%) died during follow-up and $1576(21 \%)$ died from CRC. The median follow-up time was 5.1 years (IQR: 3.4-7.5 years). 
The baseline characteristics of the study population according to red meat and processed meat intake are provided in Table 1. Patients were on average 68.9 years at diagnosis of CRC. Patients who frequently ate red meat and processed meat were more likely to smoke, they also tended to be younger, to have a higher BMI, to drink more alcohol, and to be less physically active. Family history of CRC did not differ according to red meat or processed meat intake (Table 1). Despite the observed difference among men and women in Table 1, females tended to eat less meat than men as seen in Supplementary Table 1. The higher percentages observed for females in Table 1 are mostly a function of there being more females in the included dataset than males.

In multivariable analyses among stage I-III patients, red meat and processed meat consumption in the highest versus the lowest quartile before CRC diagnosis was not associated with overall (red meat: HR:1.08, 95\%CI:0.93-1.26; processed meat: HR:1.10, 95\% CI:0.93-1.32) or CRC-specific (red meat: HR 1.09, 95\% CI:0.89-1.33; processed meat: HR:1.11, 95\% CI:0.87-1.42) survival (Table 2). No associations were observed for red meat or processed meat in the continuous analysis of servings per day for both overall and CRC-specific survival. However, stage I-III patients who reported intakes higher than the median amount of processed meat had a higher risk of death from any cause (HR:1.12,95\% CI: 1.01 -1.25), and a higher risk of CRC-specific mortality (HR:1.15, 95\% CI:0.99-1.32) compared to patients who reported eating at or less than the median. In a separate analysis among patients with stage IV CRC, no associations were observed between red meat or processed meat intake and both survival endpoints (Table 3).

No statistically significant interactions were found between red meat and processed meat and overall or CRC-specific survival with sex, cancer site, family history of CRC, BMI, or study design (Supplementary Table 2 and 3). A statistically significant interaction was observed between age and red meat intake in relation to overall survival only (pinteraction 0.003 ). Stratification by age revealed an association among those $\geq 70$ years (>median vs Smedian, HR:1.14, 95\% CI:1.00-1.30) but no association among those $<70$ years (>median vs smedian, HR:0.88, 95\% CI:0.74-1.06).

\section{Discussion}

In this large international CRC consortium, we observed no association between the highest compared with the lowest intake of red or processed meat before diagnosis and overall and CRC-specific survival. However, patients with stage I-III CRC who reported intakes higher than the study-specific median amount of processed meat had a 12\% higher risk of death compared to those who reported eating at or less than the median. We observed differences in the association between red meat and overall survival by age but no other significant effect modification was found. Higher intake of red meat (>median) was associated with worse overall survival among patients $\geq 70$ years but no association was observed among those $<70$ years.

To our knowledge only three studies have specifically examined red and processed meat in relation to CRC survival, and subsamples of two of these studies were included in the present analysis ${ }^{8,12}$.In a cohort of 2315 patients diagnosed with CRC (CPS-II cohort), red 
and processed meat intake before $\mathrm{CRC}$ diagnosis was associated with higher risk of allcause mortality (Q4vsQ1, RR:1.29, 95\% CI:1.05- 59) and cardiovascular disease (CVD) specific death (RR:1.63; 95\% CI:1.00-2.67) but not CRC-specific death (RR:1.09; 95\% CI: $0.79-1.51)^{8}$. Post-diagnostic red and processed meat intake was not associated with survival among CRC patients, however, those who consumed a higher amount both pre and post diagnosis, had a higher risk of CRC specific mortality (RR:1.79, 95\% CI:1.11 -2.89) compared to those who had consistently lower intakes. In a larger study of $3122 \mathrm{CRC}$ patients (DACHS study), red and processed meat intake was not associated with overall, CRC- specific, CVD-specific, non-CRC specific and recurrence free survival ${ }^{12}$. Similarly, in the largest study to date, among 3789 CRC patients in the European Investigation into Cancer and Nutrition (EPIC) cohort, pre-diagnostic red and processed meat intake was not associated with overall or CRC-specific survival ${ }^{11}$.

A recent systematic review and meta-analysis investigating the association between food intake and dietary patterns and overall mortality among patients with cancer, reported results with regard to meat intake (not limited to red and processed meat). Meta-analysis of four studies (including the CPS-II study and the DACHS study) revealed no significant association with overall mortality among patients with CRC when comparing the highest versus the lowest category of meat intake (RR: $1.10,95 \%$ CI: $0.84-1.43$ ), also similar to the findings of the current study ${ }^{34}$.

In this large pooled analysis of 10 epidemiological studies we did not find an association between higher red meat or processed meat intake and overall survival or CRC-specific survival, but, we found an association with overall survival and CRC- specific survival among stage I-III patients who reported eating above the median intake of processed meat compared to those who reported eating at or below the median intake. Although none of the three previous studies examined the association with meat intake when modeled as a dichotomous exposure ( $>$ median or < median intake), an analysis within the CPS-II cohort found that survivors with consistently above median red and processed meat intake both before and after CRC diagnosis had a higher risk of CRC specific mortality compared to those with consistently low intakes ${ }^{8}$ Unfortunately, we were not able to examine associations with post-diagnostic intake within the ISACC consortium. However, for some patients it is likely that temporary if not permanent dietary modifications are required after a CRC diagnosis. Limited evidence is currently available which examines dietary changes after a CRC diagnosis, although, a recent analysis of the DACHS cohort found that five years after diagnosis there was a dramatic decrease in the proportion of CRC survivors who consumed red and processed meat more than 1 time per day (from $28.7 \%$ to $2.9 \%$ ) or 1 time per day (from $37.4 \%$ to $14.5 \%)^{12}$. Future studies with several dietary measurements both before and after cancer diagnosis are necessary to evaluate and clarify the relationship between consistency in pre- and post-diagnostic meat consumption and survival.

In subgroup analyses, higher red meat intake (> median) was significantly associated with worse overall survival in patients aged $\geq 70$ years. One previous study (DACHS), of which a subset was included in this current analysis, also conducted analyses stratified by age but found no difference among survivors of CRC $\geq 70$ years or $<70$ years ${ }^{12}$. However, because of the many subgroup analyses we conducted in this analysis or because of the small group 
sizes in some subgroup analyses, the results may be due to chance. Subgroup analyses in two studies ${ }^{8,9}$ also found that among those with a family history of CRC, those with the highest meat consumption had a higher risk of death compared to those with the lowest intake, and no association was observed for those without a family history of CRC. In contrast to these findings, our large study as well as previously published results from the DACHS study, were not able to confirm these results ${ }^{12}$.

Previous research has suggested that several factors such as heterocyclic amines, N-nitroso compounds, or the use of nitrate or nitrites in the preservation of meat, may help explain the observed association between higher red and processed meat intake and risk of CRC ${ }^{35,36}$ However, there is currently no evidence as to whether increased exposure to these factors will lead to more aggressive cancers ${ }^{37}$. Furthermore, evidence on the association between red and processed meat and known molecular pathological subtypes of CRC is currently limited and inconsistent ${ }^{3839}$ More studies are necessary to evaluate novel molecular tumor subtypes associated with red and processed meat intake. Nevertheless, other lifestyle factors such as physical inactivity, and a Western dietary pattern, which often includes red and processed meat, refined grains and sugar-sweetened beverages, are associated with increased mortality risk ${ }^{37,40} \mathrm{Also}$, a lifestyle contributing to an unfavorable energy balance and hyperinsulinemia may lead to the development of more aggressive cancers ${ }^{3741,42}$.

Current dietary guidelines for cancer survivors are based on guidelines for cancer and heart disease prevention ${ }^{43}$. The findings from the current pooled analysis are an important contribution to the limited knowledge on the role of diet and CRC survival. Although our study focuses on pre-diagnostic (rather than post-diagnostic) meat consumption, and may not directly inform clinical recommendations for patients, our results suggest that meat consumption before diagnosis has no or only a weak impact on survival after CRC. There is a need for well-designed patient cohort studies focusing on red and processed meat intake after diagnosis to better inform dietary guidelines for patients with CRC.

There are a number of strengths of the present study. First, pooling studies from a large consortium with harmonized exposure and outcome resulted in the largest sample size to date to investigate associations of red and processed meat and survival after a diagnosis with CRC. Second, we were able to adjust for a wide range of potentially confounding variables. Other strengths of the present study include the comprehensive follow-up procedures carried out by each individual study which ensured thorough vital status assessment, completeness of follow-up, and a long duration of follow-up in each study. However, as this study includes harmonized data from 10 epidemiological studies, we cannot rule out differences in the completeness of follow-up between studies. We also acknowledge some limitations of our study. Among the studies included in the consortium, there were differences in dietary assessment and the ascertainment of red and processed meat intake. Some studies had much more detailed dietary information, whilst others were more limited. There were also differences between the studies in the timing of the dietary assessment due to the different study designs. However, we found no differences in overall results when stratified by original study design (i.e. cohort and case-control design). Furthermore, the studies also differed in their definitions of red and processed meat, meaning that we could not create a combined variable of red and processed meat and could therefore only analyse red meat 
(unprocessed red meat including processed red meat, i.e. sausages, in some studies) and processed meat (processed red meat and processed white meat) separately. Additionally, we did not have information harmonized on post-diagnostic dietary intake, therefore, it was not possible to assess potential changes in dietary intake which could have affected the overall results. Further limitations include the limited availability of harmonized treatment information and information on CRC recurrence. Finally, we cannot rule out the possibility of residual confounding due to unmeasured or inaccurately measured variables.

In conclusion, in the largest study to date to examine the association between pre-diagnostic red meat and processed meat intake and CRC survival, we found no significant associations between intake of red or processed meat with overall and CRC-specific survival. However, we cannot exclude a possible weak adverse association with survival for intakes higher than the study-specific median amount of red and processed meat. Future studies with dietary data at a number of time points both before and after cancer diagnosis, are required to confirm and build upon the current findings.

\section{Supplementary Material}

Refer to Web version on PubMed Central for supplementary material.

\section{Acknowledgments}

CPS-II: The authors thank the CPS-II participants and Study Management Group for their invaluable contributions to this research. The authors would also like to acknowledge the contribution to this study from central cancer registries supported through the Centers for Disease Control and Prevention National Program of Cancer Registries, and cancer registries supported by the National Cancer Institute Surveillance Epidemiology and End Results program.

DACHS: We thank all participants and cooperating clinicians, and Ute Handte-Daub, Utz Benscheid, Muhabbet Celik and Ursula Eilber for excellent technical assistance.

ISACC: The authors would like to thank all those at the ISACC Coordinating Center for helping bring together the data and people that made this project possible.

HPFS, NHS and PHS: We would like to acknowledge Qin (Carolyn) Guo and Lixue Zhu who assisted in programming for NHS and HPFS, and Haiyan Zhang who assisted in programming for the PHS. We would like to thank the participants and staff of the Nurses' Health Study and the Health Professionals Follow-Up Study, for their valuable contributions as well as the following state cancer registries for their help: AL, AZ, AR, CA, CO, CT, DE, FL, GA, ID, IL, IN, IA, KY, LA, ME, MD, MA, MI, NE, NH, NJ, NY, NC, ND, OH, OK, OR, PA, RI, SC, TN, TX, VA, WA, WY. The authors assume full responsibility for analyses and interpretation of these data.

PLCO: The authors thank Drs. Christine Berg and Philip Prorok, Division of Cancer Prevention, National Cancer Institute, the Screening Center investigators and staff or the Prostate, Lung, Colorectal, and Ovarian (PLCO) Cancer Screening Trial, Mr. Tom Riley and staff, Information Management Services, Inc., Ms. Barbara O'Brien and staff, Westat, Inc., and Drs. Bill Kopp and staff, SAIC-Frederick. Most importantly, we acknowledge the study participants for their contributions to making this study possible. The statements contained herein are solely those of the authors and do not represent or imply concurrence or endorsement by NCI.

WHI: The authors thank the WHI investigators and staff for their dedication, and the study participants for making the program possible. A full listing of WHI investigators can be found at:

http://www.whi.org/researchers/Documents\%20\%20Write\%20a\%20Paper/WHI\%20Investigator\%20Short $\% 20$ List.pdf

Funding 
ISACC: National Cancer Institute, National Institutes of Health, U.S. Department of Health and Human Services (R01 CA176272).

GECCO: National Cancer Institute, National Institutes of Health, U.S. Department of Health and Human Services (U01 CA137088; R01 CA059045).

CCFR: This work was supported by grant UM1 CA167551 from the National Cancer Institute and through cooperative agreements with the following CCFR centers: Seattle Colorectal Cancer Family Registry (U01/U24 CA074794).

CPS-II: The American Cancer Society funds the creation, maintenance, and updating of the Cancer Prevention Study-II (CPS-II) cohort. This study was conducted with Institutional Review Board approval.

DACHS: German Research Council (Deutsche Forschungsgemeinschaft, BR 1704/6-1, BR 1704/6-3, BR 1704/6-4 and CH 117/1-1), and the German Federal Ministry of Education and Research (01KH0404, 01ER0814, 01ER1505A, 01ER1505B).

DALS: National Institutes of Health (R01 CA48998 to M. L. Slattery).

HPFS is supported by the National Institutes of Health (P01 CA 055075, UM1 CA167552, U01 CA167552, R01 CA137178, R01 CA151993, R35 CA197735, and P50 CA127003), NHS by the National Institutes of Health (UM1 CA186107, R01 CA137178, P01 CA87969, R01 CA151993, R35 CA197735, and P50 CA127003) and PHS by the National Institutes of Health (R01 CA042182).

PLCO: Intramural Research Program of the Division of Cancer Epidemiology and Genetics and supported by contracts from the Division of Cancer Prevention, National Cancer Institute, NIH, DHHS.

VITAL: National Institutes of Health (K05 CA154337).

WHI: The WHI program is funded by the National Heart, Lung, and Blood Institute, National Institutes of Health, U.S. Department of Health and Human Services through contracts HHSN268201100046C, HHSN268201100001C, HHSN268201100002C, HHSN268201100003C, HHSN268201100004C, and HHSN271201100004C.

\section{References}

1. World Cancer Research Fund/American Institute for Cancer Research. Continuous Update Project Report: Diet, Nutrition, Physcial Activity and Colorectal Cancer. 2017.

2. Bouvard V, Loomis D, Guyton KZ, et al. Carcinogenicity of consumption of red and processed meat. Lancet Oncol 2015;16:1599-600. [PubMed: 26514947]

3. Chan DS, Lau R, Aune D, et al. Red and processed meat and colorectal cancer incidence: metaanalysis of prospective studies. PLoS One $2011 ; 6:$ e20456. [PubMed: 21674008]

4. Carr PR, Walter V, Brenner H, et al. Meat subtypes and their association with colorectal cancer: Systematic review and meta-analysis. Int J Cancer 2016; 138:293-302. [PubMed: 25583132]

5. van Meer S, Leufkens AM, Bueno-de-Mesquita HB, et al. Role of dietary factors in survival and mortality in colorectal cancer: a systematic review. Nutr Rev 2013;71:631-41. [PubMed: 24032367]

6. Vrieling A, Kampman E. The role of body mass index, physical activity, and diet in colorectal cancer recurrence and survival: a review of the literature. Am J Clin Nutr 2010;92:471-90. [PubMed: 20729339]

7. Dray X, Boutron-Ruault MC, Bertrais S, et al. Influence of dietary factors on colorectal cancer survival. Gut 2003;52:868-73. [PubMed: 12740344]

8. McCullough ML, Gapstur SM, Shah R, et al. Association between red and processed meat intake and mortality among colorectal cancer survivors. J Clin Oncol 2013;31:2773-82. [PubMed: 23816965]

9. Zell JA, Ignatenko NA, Yerushalmi HF, et al. Risk and risk reduction involving arginine intake and meat consumption in colorectal tumorigenesis and survival. Int J Cancer 2007;120:459-68. [PubMed: 17096347]

10. Zell JA, Ziogas A, Bernstein L, et al. Meat consumption, nonsteroidal anti-inflammatory drug use, and mortality among colorectal cancer patients in the California Teachers Study. Cancer Prev Res (Phila) 2010;3:865-75. [PubMed: 20551290] 
11. Ward HA, Norat T, Overvad K, et al. Pre-diagnostic meat and fibre intakes in relation to colorectal cancer survival in the European Prospective Investigation into Cancer and Nutrition. Br J Nutr 2016;116:316-25. [PubMed: 27193442]

12. Carr PR, Jansen L, Walter V, et al. Associations of red and processed meat with survival after colorectal cancer and differences according to timing of dietary assessment. Am J Clin Nutr 2016;103:192-200. [PubMed: 26607936]

13. Calle EE, Rodriguez C, Jacobs EJ, et al. The American Cancer Society Cancer Prevention Study II Nutrition Cohort: rationale, study design, and baseline characteristics. Cancer 2002;94:2490-501. [PubMed: 12015775]

14. Rimm EB, Giovannucci EL, Willett WC, et al. Prospective study of alcohol consumption and risk of coronary disease in men. Lancet 1991;338:464-8. [PubMed: 1678444]

15. Belanger CF, Hennekens CH, Rosner B, et al. The nurses' health study. Am J Nurs 1978;78:103940. [PubMed: 248266]

16. Belanger C, Speizer FE, Hennekens CH, et al. The nurses' health study: current findings. Am J Nurs 1980;80:1333. [PubMed: 6901582]

17. Colditz GA, Manson JE, Hankinson SE. The Nurses' Health Study: 20-year contribution to the understanding of health among women. J Womens Health 1997;6:49-62. [PubMed: 9065374]

18. Final report on the aspirin component of the ongoing Physicians' Health Study. Steering Committee of the Physicians' Health Study Research Group. N Engl J Med 1989;321:129-35. [PubMed: 2664509]

19. Prorok PC, Andriole GL, Bresalier RS, et al. Design of the Prostate, Lung, Colorectal and Ovarian (PLCO) Cancer Screening Trial. Control Clin Trials 2000;21:273s-309s. [PubMed: 11189684]

20. Gohagan JK, Prorok PC, Hayes RB, et al. The Prostate, Lung, Colorectal and Ovarian (PLCO) Cancer Screening Trial of the National Cancer Institute: history, organization, and status. Control Clin Trials 2000;21:251s-272s. [PubMed: 11189683]

21. White E, Patterson RE, Kristal AR, et al. VITamins And Lifestyle cohort study: study design and characteristics of supplement users. Am J Epidemiol 2004;159:83-93. [PubMed: 14693663]

22. Design of the Women's Health Initiative clinical trial and observational study. The Women's Health Initiative Study Group. Control Clin Trials 1998;19:61-109. [PubMed: 9492970]

23. Brenner H, Chang-Claude J, Seiler CM, et al. Protection from colorectal cancer after colonoscopy: a population-based, case-control study. Ann Intern Med 2011;154:22-30. [PubMed: 21200035]

24. Lilla C, Verla-Tebit E, Risch A, et al. Effect of NAT1 and NAT2 genetic polymorphisms on colorectal cancer risk associated with exposure to tobacco smoke and meat consumption. Cancer Epidemiol Biomarkers Prev 2006;15:99-107. [PubMed: 16434594]

25. Slattery ML, Potter J, Caan B, et al. Energy balance and colon cancer-- beyond physical activity. Cancer Res 1997;57:75-80. [PubMed: 8988044]

26. Peters U, Hutter CM, Hsu L, et al. Meta-analysis of new genome-wide association studies of colorectal cancer risk. Hum Genet 2012;131:217-34. [PubMed: 21761138]

27. Peters U, Jiao S, Schumacher FR, et al. Identification of Genetic Susceptibility Loci for Colorectal Tumors in a Genome-Wide Meta-analysis. Gastroenterology 2013; 144:799-807.e24. [PubMed: 23266556]

28. Hutter CM, Chang-Claude J, Slattery ML, et al. Characterization of gene-environment interactions for colorectal cancer susceptibility loci. Cancer Res 2012;72:2036-44. [PubMed: 22367214]

29. Chan AT, Ogino S, Fuchs CS. Aspirin use and survival after diagnosis of colorectal cancer. Jama 2009;302:649-58. [PubMed: 19671906]

30. Curb JD, McTiernan A, Heckbert SR, et al. Outcomes ascertainment and adjudication methods in the Women's Health Initiative. Ann Epidemiol 2003;13:S122-8. [PubMed: 14575944]

31. Miller AB, Yurgalevitch S, Weissfeld JL. Death review process in the Prostate, Lung, Colorectal and Ovarian (PLCO) Cancer Screening Trial. Control Clin Trials 2000;21:400s-406s. [PubMed: 11189691]

32. Hoffmeister M, Jansen L, Rudolph A, et al. Statin use and survival after colorectal cancer: the importance of comprehensive confounder adjustment. J Natl Cancer Inst 2015;107:djv045. [PubMed: 25770147] 
33. Schemper M, Smith TL. A note on quantifying follow-up in studies of failure time. Control Clin Trials 1996;17:343-6. [PubMed: 8889347]

34. Schwedhelm C, Boeing H, Hoffmann G, et al. Effect of diet on mortality and cancer recurrence among cancer survivors: a systematic review and meta-analysis of cohort studies. Nutr Rev 2016;74:737-748. [PubMed: 27864535]

35. Bastide NM, Pierre FH, Corpet DE. Heme iron from meat and risk of colorectal cancer: a metaanalysis and a review of the mechanisms involved. Cancer Prev Res (Phila) $2011 ; 4: 177-84$. [PubMed: 21209396]

36. Cross AJ, Sinha R. Meat-related mutagens/carcinogens in the etiology of colorectal cancer. Environ Mol Mutagen 2004;44:44-55. [PubMed: 15199546]

37. Lee J, Jeon JY, Meyerhardt JA. Diet and lifestyle in survivors of colorectal cancer. Hematol Oncol Clin North Am 2015;29:1-27. [PubMed: 25475570]

38. Carr PR, Jansen L, Bienert S, et al. Associations of red and processed meat intake with major molecular pathological features of colorectal cancer. Eur J Epidemiol 2017;32:409-418. [PubMed: 28646407]

39. Carr PR, Alwers E, Bienert S, et al. Lifestyle factors and risk of sporadic colorectal cancer by microsatellite instability status: a systematic review and meta $\neg$ analyses. Ann Oncol 2018;29:825834. [PubMed: 29438474]

40. Meyerhardt JA, Niedzwiecki D, Hollis D, et al. Association of dietary patterns with cancer recurrence and survival in patients with stage III colon cancer. Jama 2007;298:754-64. [PubMed: 17699009]

41. Morikawa T, Kuchiba A, Yamauchi M, et al. Association of CTNNB1 (beta- catenin) alterations, body mass index, and physical activity with survival in patients with colorectal cancer. Jama 2011 ; 305:1685-94. [PubMed: 21521850]

42. Morikawa T, Kuchiba A, Lochhead P, et al. Prospective analysis of body mass index, physical activity, and colorectal cancer risk associated with beta-catenin (CTNNB1) status. Cancer Res 2013;73:1600-10. [PubMed: 23442321]

43. Rock CL, Doyle C, Demark-Wahnefried W, et al. Nutrition and physical activity guidelines for cancer survivors. CA Cancer J Clin 2012;62:243-74. [PubMed: 22539238] 
What You Need To Know

\section{Background}

Red and processed meat intake is associated with colorectal cancer (CRC) incidence. We investigated whether intake is associated with patient survival after diagnosis.

\section{Findings}

In this large pooled consortium, intake of red and processed meat before a diagnosis of $\mathrm{CRC}$ was not associated with shorter survival time after diagnosis, although a weak adverse association cannot be excluded.

\section{Implications for patient care}

The findings from the current pooled analysis suggest no relevant impact of red and processed meat intake before diagnosis on CRC survival. Further studies are needed of diets before and after CRC diagnosis. 
Table 1.

Baseline characteristics of study participants according to red meat and processed meat intake ${ }^{l}$.

\begin{tabular}{|c|c|c|c|c|c|c|c|c|c|c|}
\hline & \multicolumn{4}{|c|}{ Red meat(servings/day) ${ }^{2}$} & \multirow[b]{2}{*}{$\mathbf{P}^{*}$} & \multicolumn{4}{|c|}{ Processed meat (servings/day) ${ }^{3}$} & \multirow[b]{2}{*}{$\mathbf{P}^{*}$} \\
\hline & $\begin{array}{c}\mathbf{Q 1} \\
(\mathbf{n}=\mathbf{2 1 2 0})\end{array}$ & $\begin{array}{c}\mathbf{Q 2} \\
(\mathrm{n}=\mathbf{2 0 7 3})\end{array}$ & $\begin{array}{c}\mathbf{Q 3} \\
(\mathrm{n}=1911)\end{array}$ & $\begin{array}{c}\text { Q4 } \\
(n=1523)\end{array}$ & & $\begin{array}{c}Q 1 \\
(n=1493)\end{array}$ & $\begin{array}{c}\mathbf{Q 2} \\
(\mathrm{n}=2846)\end{array}$ & $\begin{array}{c}\mathbf{Q 3} \\
(\mathrm{n}=1929)\end{array}$ & $\begin{array}{c}\mathbf{Q 4} \\
(\mathrm{n}=1080)\end{array}$ & \\
\hline \multirow[t]{2}{*}{ Age at diagnosis, mean (SD) } & & & & & $<.001$ & & & & & $<.001$ \\
\hline & $69.8(9.3)$ & $69.3(9.2)$ & $69.2(9.5)$ & $67.6(9.9)$ & & $69.9(8.8)$ & $69.3(9.9)$ & $69.1(9.5)$ & $68.3(9.6)$ & \\
\hline Sex, n (\%) & & & & & $<.001$ & & & & & $<.001$ \\
\hline Male & $884(42)$ & $1120(54)$ & $850(44)$ & $587(39)$ & & $843(56)$ & $1129(40)$ & $958(49)$ & $506(47)$ & \\
\hline Female & $1236(58)$ & $953(46)$ & $1061(56)$ & $936(61)$ & & $650(44)$ & $1717(60)$ & $971(51)$ & $574(53)$ & \\
\hline \multirow[t]{2}{*}{ BMI, $\mathbf{k g m}^{2}$ (SD) } & & & & & $<.001$ & & & & & $<.001$ \\
\hline & $26.4(4.4)$ & $27.2(4.3)$ & $27.6(4.8)$ & $28.6(5.4)$ & & $26.6(4.3)$ & $27.0(4.3)$ & $27.7(4.8)$ & $28.6(5.7)$ & \\
\hline Ever smoker, n (\%) & & & & & 0.004 & & & & & $<.001$ \\
\hline No & $961(45)$ & $842(41)$ & $823(43)$ & $616(40)$ & & $641(43)$ & $1328(47)$ & $767(40)$ & $384(35)$ & \\
\hline Yes & $1148(54)$ & $1228(59)$ & $1079(56)$ & $901(59)$ & & $841(56)$ & $1511(53)$ & $1156(60)$ & $691(64)$ & \\
\hline Alcohol, n (\%) & & & & & $<.001$ & & & & & $<.001$ \\
\hline Non drinker & $911(43)$ & $743(36)$ & $710(37)$ & $616(40)$ & & $670(45)$ & $1136(40)$ & $712(37)$ & $459(43)$ & \\
\hline $1-28 g / d a y$ & $877(41)$ & $958(46)$ & $857(45)$ & $643(42)$ & & $641(43)$ & $1346(47)$ & $883(46)$ & $463(43)$ & \\
\hline$>28 g /$ day & $191(9)$ & $297(14)$ & $294(15)$ & $209(14)$ & & $176(12)$ & $336(12)$ & $324(17)$ & $155(14)$ & \\
\hline Education, $\mathrm{n}(\%)$ & & & & & $<.001$ & & & & & $<.001$ \\
\hline Less than high school & $250(12)$ & $211(10)$ & $270(14)$ & $176(12)$ & & $92(6)$ & $458(16)$ & $228(12)$ & $108(10)$ & \\
\hline High school graduate & $684(32)$ & $754(36)$ & $648(34)$ & $459(30)$ & & $437(29)$ & $1024(36)$ & $683(35)$ & $319(30)$ & \\
\hline Some college/technical school & $440(21)$ & $469(23)$ & $418(22)$ & $374(25)$ & & $420(28)$ & $438(15)$ & $424(22)$ & $329(30)$ & \\
\hline College graduate & $736(35)$ & $634(31)$ & $572(30)$ & $512(34)$ & & $539(36)$ & $916(32)$ & $591(31)$ & $322(30)$ & \\
\hline Family history of CRC, n (\%) & & & & & 0.25 & & & & & 0.23 \\
\hline No & $1708(81)$ & $1682(81)$ & $1510(79)$ & $1179(77)$ & & $1173(79)$ & $2308(81)$ & $1511(78)$ & $824(76)$ & \\
\hline Yes & $303(14)$ & 295 (14) & $307(16)$ & $233(15)$ & & $206(14)$ & $430(15)$ & $310(16)$ & $175(16)$ & \\
\hline Regular aspirin/NSAID use, $\mathrm{n}(\%$ & & & & & 0.09 & & & & & 0.004 \\
\hline No & $1476(70)$ & $1427(69)$ & $1299(68)$ & $1007(66)$ & & $992(66)$ & $1994(70)$ & $1300(67)$ & 707 (65) & \\
\hline Yes & $620(29)$ & $624(30)$ & $594(31)$ & $505(33)$ & & $489(33)$ & 817 (29) & $606(31)$ & $368(34)$ & \\
\hline History of diabetes, n (\%) & & & & & 0.03 & & & & & 0.01 \\
\hline No & $1609(76)$ & $1543(74)$ & $1383(72)$ & $1068(70)$ & & $1058(71)$ & $2216(78)$ & $1395(72)$ & $696(64)$ & \\
\hline Yes & $195(9)$ & $222(11)$ & $229(12)$ & $145(10)$ & & $117(8)$ & $324(11)$ & $223(12)$ & $86(8)$ & \\
\hline Physical activity ${ }^{4}, \mathrm{n}(\%)$ & & & & & $<.001$ & & & & & $<.001$ \\
\hline No & $315(15)$ & $355(17)$ & $370(19)$ & $325(21)$ & & $302(20)$ & 397 (14) & $374(19)$ & $292(27)$ & \\
\hline Yes & $1393(66)$ & $1342(65)$ & $1187(62)$ & $835(55)$ & & $1001(67)$ & $1931(68)$ & $1227(64)$ & $598(55)$ & \\
\hline
\end{tabular}

Clin Gastroenterol Hepatol. Author manuscript; available in PMC 2020 July 01. 


\begin{tabular}{|c|c|c|c|c|c|c|c|c|c|c|}
\hline & \multicolumn{4}{|c|}{ Red meat(servings/day) ${ }^{2}$} & \multirow[b]{2}{*}{$\mathbf{P}^{*}$} & \multicolumn{4}{|c|}{ Processed meat (servings/day) ${ }^{3}$} & \multirow[b]{2}{*}{$\mathbf{P}^{*}$} \\
\hline & $\begin{array}{c}\mathbf{Q 1} \\
(\mathbf{n}=\mathbf{2 1 2 0})\end{array}$ & $\begin{array}{c}\mathbf{Q 2} \\
(\mathrm{n}=2073)\end{array}$ & $\begin{array}{c}\text { Q3 } \\
(n=1911)\end{array}$ & $\begin{array}{c}\text { Q4 } \\
(n=1523)\end{array}$ & & $\begin{array}{c}Q 1 \\
(n=1493)\end{array}$ & $\begin{array}{c}Q 2 \\
(n=2846)\end{array}$ & $\begin{array}{c}\text { Q3 } \\
(n=1929)\end{array}$ & $\begin{array}{c}\text { Q4 } \\
(n=1080)\end{array}$ & \\
\hline Tumor site, $\mathbf{n}(\%)$ & & & & & $<.001$ & & & & & $<.001$ \\
\hline Proximal colon & 929 (44) & $915(44)$ & $799(42)$ & $665(44)$ & & $679(45)$ & $1195(42)$ & $794(41)$ & $502(46)$ & \\
\hline Distal colon & $621(29)$ & $580(28)$ & $578(30)$ & $522(34)$ & & $454(30)$ & $811(28)$ & $597(31)$ & $372(34)$ & \\
\hline Rectum & $526(25)$ & $542(26)$ & $498(26)$ & $311(20)$ & & $328(22)$ & $788(28)$ & $510(26)$ & $186(17)$ & \\
\hline Unknown & $44(2)$ & $36(2)$ & $36(2)$ & $25(2)$ & & $32(2)$ & $52(2)$ & $28(1)$ & $20(2)$ & \\
\hline Stage of CRC, n (\%) & & & & & 0.27 & & & & & $<.001$ \\
\hline I & $624(29)$ & $584(28)$ & $586(31)$ & $465(31)$ & & $468(31)$ & $746(26)$ & $556(29)$ & $361(33)$ & \\
\hline II/III & $1120(53)$ & $1150(55)$ & $1030(54)$ & $784(51)$ & & $747(50)$ & $1634(57)$ & $1060(55)$ & $521(48)$ & \\
\hline IV & $272(13)$ & $255(12)$ & $214(11)$ & $189(12)$ & & $192(13)$ & 347 (12) & $230(12)$ & $134(12)$ & \\
\hline
\end{tabular}

${ }^{1}$ Numbers of participants do not always equal total numbers because of missing values for some variables.

2

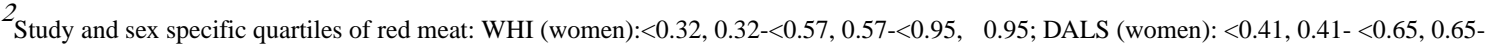
$<0.95, \searrow 0.95$; DALS (men): $<0.66,0.66-<1.12,1.12-<1.78, \geq 1.78$; PMH (women): $<0.29,0.29-<0.43,0.43-<0.57, \searrow 0.57$; VITAL (women): <0.23, $0.23-<0.48,0.48-<0.77, \geq 0.77$; VITAL (men): $<0.37,0.37-<0.68,0.68-<1.05, \geq 1.05 ;$ PLCO (women): $<0.38,0.38-<0.62,0.62-<0.89, \geq 0.89$; PLCO (men): $<0.7,0.7-<1.25,1.25-<1.8, \geq 1.8$; DACHS (women): $<0.42,0.42-<0.64,0.64-<0.84, \geq 0.84$; DACHS (men): $<0.63,0.63-<0.84,0.84-<1.06$, $\geq 1.06$; HPFS (men): $<0.41,0.41-<0.73,0.73-<1.31, \geq 1.31$; PHS (men): $<0.46,0.46-<0.56,0.56-<0.86, \geq 0.86$; NHS (women): $<0.34,0.34-<0.62$, $0.62-<1.08, \geq 1.08$; CPS2 (women): $<0.3,0.3-<0.48,0.48-<0.79, \geq 0.79$; CPS2 (men): $<0.45,0.45-<0.70,0.70-<1.12, \geq 1.12$.

${ }^{3}$ Study and sex specific quartiles of processed meat: WHI (women): $<0.07,0.07-<0.16,0.16-<0.36, \geq 0.36$; DALS (women): $<0.05,0.05-<0.11,0.11$ $-<0.21, \searrow 0.21$; DALS (men): $<0.11,0.11-<0.24,0.24-<0.44, \searrow 0.44$; VITAL (women): <0.04, 0.04-<0.14, 0.14-<0.28, У0.28; VITAL (men): <0.14, $0.14-<0.27,0.27-<0.54, \searrow 0.54$; PLCO (women): $<0.05,0.05-<0.10,0.10-<0.19, \searrow 0.19$; PLCO (men): $<0.12,0.12-<0.25,0.25-<0.49, \geq 0.49$; DACHS (women): $<0.42,0.42-<0.84,0.84, \geq 0.84$; DACHS (men): $<0.64,0.64-<0.84,0.84-<1.28, \geq 1.28$; HPFS (men): $0,0-<0.04,0.04-<0.07$, $\searrow 0.07$; PHS (men): $<0.09,0.09-<0.20,0.20-<0.26, \geq 0.26$; NHS (women): $0,>0-<0.07,0.07,>0.07$; CPS2 (women): $<0.08,0.08-<0.15,0.15-$ $<0.25, \searrow 0.25$; CPS2 (men): $<0.15,0.15-<0.27,0.27-<0.61, \searrow 0.61$

4 Physical activity: no, vigorous and moderate physical activity < 1 hour/week; yes, vigorous and moderate physical activity $\geq 1$ hour/week.

P value for differences in frequencies across meat strata

Abbreviations: BMI, body mass index; CI, confidence interval; CRC, colorectal cancer; HR, hazard ratio; NSAID, non steroidal antiinflammatory drug; SD, standard deviation

Clin Gastroenterol Hepatol. Author manuscript; available in PMC 2020 July 01. 


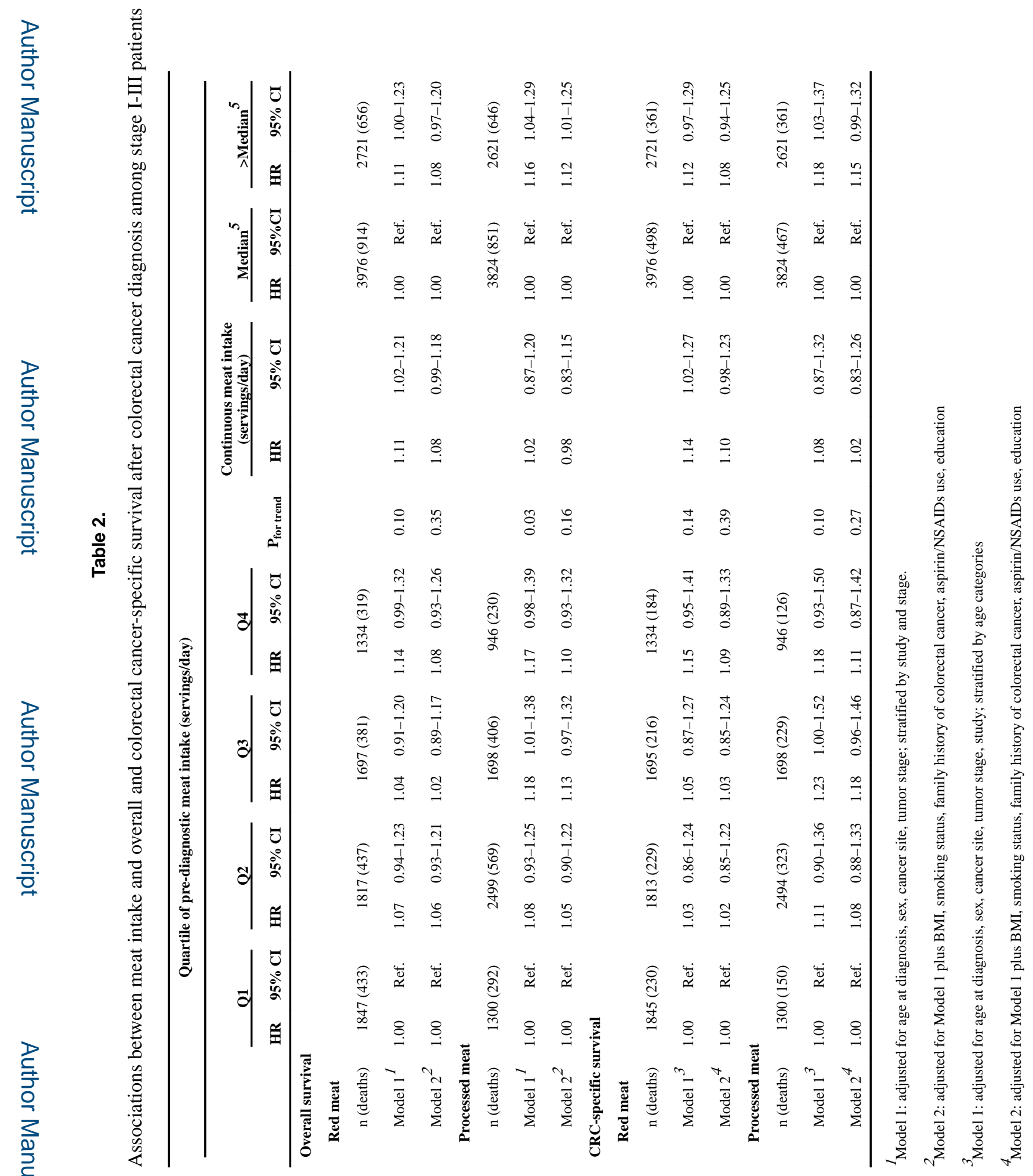

Clin Gastroenterol Hepatol. Author manuscript; available in PMC 2020 July 01. 


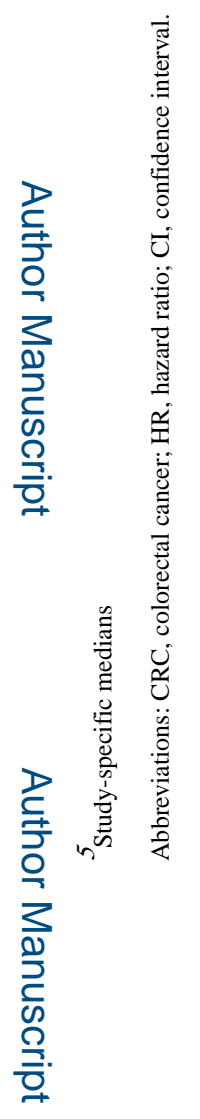

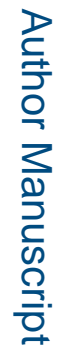

롤

Clin Gastroenterol Hepatol. Author manuscript; available in PMC 2020 July 01. 


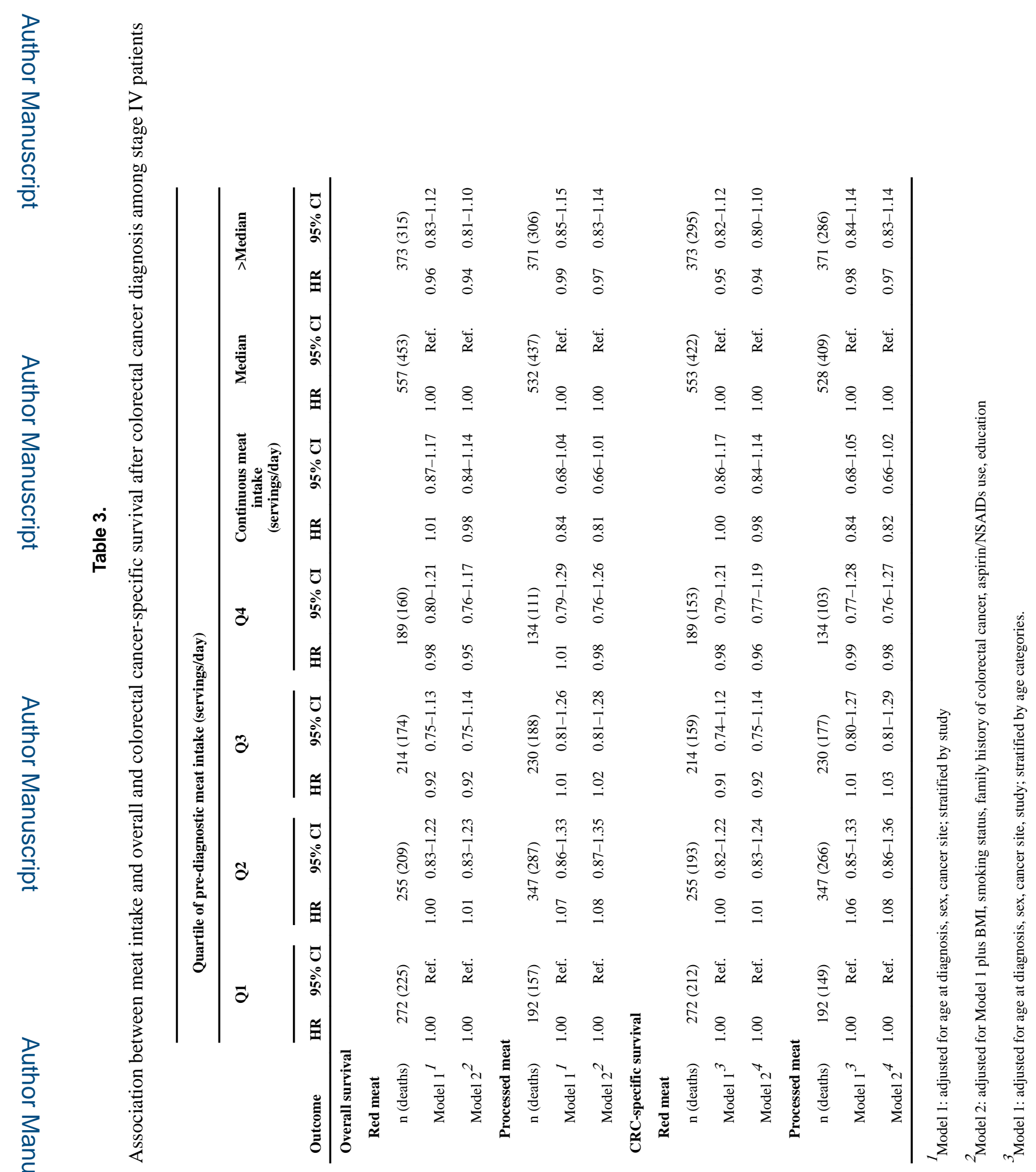

Clin Gastroenterol Hepatol. Author manuscript; available in PMC 2020 July 01. 


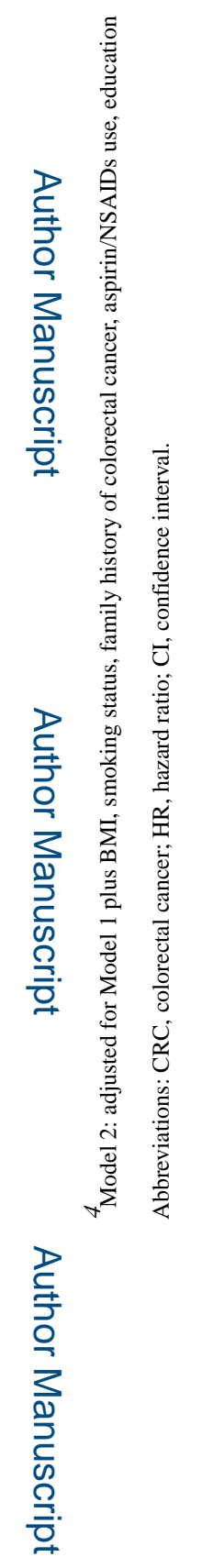

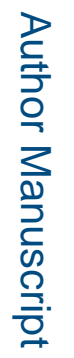

Clin Gastroenterol Hepatol. Author manuscript; available in PMC 2020 July 01. 